\title{
Feasibility and Validity of the Daily Physical Pain Intensity (DPPI) Scale for Pain Assessment
}

\author{
Yeon-Gyo Nam, Dong-Yeop Lee, Jae-Ho Yu, Jin-Seop Kim, Ji-heon Hong \\ Department of Physical Therapy, Graduate School of Sun Moon University, Asan, Korea
}

Purpose: The purpose of this study was the examination of the correlations between the daily physical pain intensity (DPPI) scale and the numeric rating scale (NRS), and between the DPPI scale and the quality of life (OOL) of short form health survey (SF-36) and beck depression inventory (BDI) questionnaires. The focus of the DPPI scale was the daily-living activities of the individual, and the scale contains three items regarding the pain per movement range, the pain per day, and the pain when touching the pain area.

Methods: A total of 241 adults answered the DPPI, NRS, SF-36, and BDI questionnaires. Pearson's correlation coefficients were calculated for the various relations of the DPPI to the other scales.

Results: High correlations were shown between the NRS and the DPPI $(r=0.809, p<0.05)$. The DPPI scale $(r=0.437, p<0.05)$ showed "moderate" significant correlations with the SF-36 and the NRS $(r=0.370, p<0.05)$, and it showed "weak" significant correlations with the SF-36. There are no statistically significant correlations between the DPPI, the NRS score, and the BDI score ( $p>0.05)$.

Conclusion: This study was the first attempt to establish the concurrent validity of a new focus on daily-living activities for the assessment of pain. This study showed promise for the development of activities of daily living focused tool for an assessment of the subjective pain in patients that was more objective.

Keywords: Pain, Pain measurement, Musculoskeletal pain

\section{INTRODUCTION}

Pain is defined as an unpleasant sensory and emotional experience that is associated with actual or potential tissue damage, or is described in terms of such damage. ${ }^{1}$ In clinical examinations, pain is most-often measured using a visual analog scale (VAS) to capture the pain intensity; however, other complex elements of pain are ignored by the VAS because of the scale's simplicity. ${ }^{2}$ Pain is often accompanied by substantial disability, depression, and anxiety. ${ }^{3}$

For the measurement of a patient's overall disability in the activities of daily living and the cause of the pain, the various aspects of pain must be considered. First, joint pain is associated with a range of motions and unsuccessful movement. ${ }^{4}$ In the shoulder joint, glenohumeral ligaments contribute static stabilizers in the end-range of motion; however, since the joint is not stabilized by isometric articular ligaments, stability in the mid-range must be achieved by

Received Nov 17, 2016 Revised Dec 17, 2016

Accepted Dec 20, 2016

Corresponding author Ji-heon Hong

E-mail hgh1020@hanmail.net mechanisms other than capsuloligamentous restraints. ${ }^{5}$ In this way, as specific muscles and ligaments work, the pain varies with each range of motion. A previous study supported a relationship between the disability that is due to pain-associated LOM and work dimensions. ${ }^{6}$ Second, the authors considered the length of time that pain persists over a day. The level of physical activity varied both between people and for an individual across different times and days. ${ }^{7}$ Continuous pain during the day interrupts physical activities and has emotional qualities. ${ }^{89}$ The other issue is the sensitivity to pain. Pain sensitivity is well correlated with the severity of back pain and/or leg pain in patients with lumbar spinal stenosis. ${ }^{10}$ The influence of pain sensitivity on treatment outcomes is a critical concern. ${ }^{11}$ Many studies showed that a strong relationship between pain sensitivity and physical activity. ${ }^{12}$ But in clinical settings, along with the pain intensity, one of the most-common assessments is that for the pain location and extent ("Tell me where are you have pain?"). In chronic-

Copylight (C2016 The Korea Society of Physical Therapy

This is an Open Access article distribute under the terms of the Creative Commons Attribution Non-commercial License (Http:// creativecommons.org/license/by-nc/4.o.) which permits unrestricted non-commercial use, distribution, and reproduction in any medium, provided the original work is properly cited. 
pain patients, a relationship has been observed between the disability experienced in daily activities and the severity of pain. ${ }^{13}$ A previous study supported a relationship between disability and both pain and work dimensions, ${ }^{4}$ and pain negatively impacts upon the person experiencing it, affecting his/her social functioning and physical and psychological wellbeing. ${ }^{14}$ However, these pain-assessment tools, like a proxy pain questionnaire, were scored only for presence and absence, ${ }^{15}$ and such simple tools may not reflect the actual pain severity that is associated with the activities of daily living (ADL). ${ }^{5}$ In chronic-pain patients, a variable relationship was observed between the disability experienced in daily activities and the severity of pain. ${ }^{13}$

A new assessment tool was therefore required whereby the range, duration, and sensitivity of the pain are included. A pain-assessment tool that is clinically systematic and relevant to individuals with physical pain focuses on the ADL, and the developed scale contains the three aspects of pain, as follows: "How is the pain when the joint is moving? Especially the starting range, mid-range, and final range of the movement." "When you are resting how long does the pain persist for? Divide one day into morning, afternoon, and night pains." "How sensitive is the pain area? Each item can be checked with a number from 0 to 10 according to the severity." The purpose of this study was the examination of the correlations between the DPPI scale and the NRS, and between the DPPI scale and the questionnaires regarding ADL disability and depression (BDI).

\section{METHODS}

\section{Subjects}

This study was conducted on 241 subjects aged 20 years to 65

Table 1. General characteristics of subjects

$(n=73)$

\begin{tabular}{llrr}
\hline & Item & $\mathrm{N}(\%)$ & Mean \pm SD \\
\hline Age (year) & & & $27.86 \pm 10.249$ \\
Sex & Male & $29(39.7)$ & \\
& Female & $44(60.3)$ & \\
Height $(\mathrm{cm})$ & & & $166.94 \pm 8.019$ \\
Weight $(\mathrm{kg})$ & & & \\
Pain area & Shoulder & $22(30.1)$ & \\
& Lumbar & $23(31.5)$ & \\
& Neck & $8(11.0)$ & \\
& Knee & $8(11.0)$ & \\
& Etc & $12(16.4)$ & \\
\hline
\end{tabular}

years who experienced pain, and no particular disease was targeted. Of all of the subjects, 94 were male and 147 were female, with an average age of $25.1 \pm 7.4$ years. The authors wanted to exclude the cases where various personal matters like relationship problems with friends or family influenced quality-of-life (QOL) changes, so only those people who answered "Yes" to the question about pain-based difficulties regarding ADL were selected. The screened data are from a total of 73 subjects that comprise 29 males and 44 females (Table 1).

\section{Measurement}

All of the subjects were fully informed about the procedures and aims of the current experiment, and the study protocol was approved by the authors' institutional review board from Sun-moon University (SM201512-022). The authors randomly asked passersby to complete a questionnaire if they had any physical pain. After the authors explained the purpose and objective of the present study to the subjects and ask the following questions

\section{1) Daily physical pain intensity (DPPI)}

To the subjects, the following questions were asked: How is the pain when the joint is moving? Especially the starting range, midrange, and final range of the movement. When you are resting, how long does the pain persist for? Divide one day into morning, afternoon, and night pains. What is the pain like when you press the pain area?

\section{(1) Definition and scoring}

I. Movement pain: Check the pain when the patient is moving their joint during daily living. See if the purpose of the movement can be remembered or not. Separate the movement into three ranges (starting range, mid-range, and end range). Each item can be checked with a number from 0 to 10 according to the severity, where 0 is no pain at all and 10 represents the highest imaginable level of pain possible.

A. Starting-range pain - The pain of the start range and any pain occurring during the movement. If the patient cannot move their joint or segments due to severe pain, check the start pain and score all of the remaining items about movement as 10 .

B. Mid-range pain - The pain of the mid-range of the movement. The range between the starting range and the end range. 
C. End-range pain - The pain of the end range of the movement. Although the movement is not successful because of pain, the "end range" indicates the final range of the patient's performance.

II. Rest pain: Check the pain when the patient is not moving their joint in daily living. Rest pain usually occurs in the extremities, and during rest periods in the sitting or lying position. Separate one day into three periods (morning, afternoon, and night). If pain is felt all day, none of the items are scored as 0 . Each item can be checked with a number from 0 to 10 according to the severity, where 0 is no pain at all and 10 represents the highest imaginable level of pain possible.

A. Morning (after waking up) - The pain of the morning. Originally, the term referred to sunrise or waking up. For individuals, this is applicable to the intervals between their sleep and activity times.

B. Afternoon (activity time) - The pain of the afternoon. Afternoon is the time between morning and night, or refers to the peak of activity.

C. Night (before sleep) - The pain of night. Night is the period of time before sleep. Can the patient recall the pain from when they are lying on their bed?

III. Pressure/Touch pain: The pain when the pain area is pressed with a finger. Press until the distal end of the nail color starts to change to white. If the patient has too-much pain before the nailcolor change, check number 10. In the case of myofascial pain syndrome (MPS), also known as chronic myofascial pain (CMP), replace the application of pressure with a light touch. Each item can be checked with a number from 0 to 10 according to the severity, where 0 is no pain at all and 10 represents the highest imaginable level of pain possible.

\section{2) NRS}

The Numeric Rating Scale (NRS-11) is an 11-point scale for patient self-reporting of pain. It is for adults and children 10 years old or older. 0 is No Pain, 1-3 is Mild Pain (nagging, annoying, interfering little with ADLs), 4-6 is Moderate Pain (interferes significantly with ADLs), 7-10 is Severe Pain (disabling; unable to perform ADLs)

\section{3) SF-36}

The SF-36 consists of eight scaled scores, which are the weighted sums of the questions in their section. Each scale is directly transformed into a $0-100$ scale on the assumption that each question carries equal weight. The lower the score means more disability. The higher the score the less disability i.e., a score of zero is equivalent to maximum disability and a score of 100 is equivalent to no disability. To calculate the scores it is necessary to purchase special software. Pricing depends on the number of scores that the researcher needs to calculate. The eight sections are vitality, physical functioning, bodily pain, general health perceptions, physical role functioning, emotional role functioning, social role functioning and mental health.

\section{4) BDI}

The Beck Depression Inventory (BDI) is a 21-item, self-report rating inventory that measures characteristic attitudes and symptoms of depression. The BDI takes approximately 10 minutes to complete, although clients require a fifth-sixth grade reading level to adequately understand the questions.

\section{Statistical analysis}

Statistical analyses were calculated for the various relations of the DPPI to the other scales using SPSS Ver. 12.0 for Windows (SPSS Inc., USA) and a significance level of $\mathrm{p}=0.05$. The correlations among the preliminary data were measured using Pearson's correlation. In this study, correlation is an effect size, and the strength of the correlation can therefore be verbally described using the guide that Evans (1996) suggested for the absolute values, as follows: $r$ : 0.00-0.19 “very weak", 0.20-0.39 “weak", 0.40-0.59 “moderate”, 0.600.79 "strong", and 0.80-1.0 "very strong." These values were deemed to be statistically significant. The demographic data and scores for the questionnaires are expressed in the form of the mean \pm standard deviation.

\section{RESULTS}

The mean of the DPPI score for the pain assessment is $18.70 \pm$ 10.84, while those for the NRS, SF-36, and BDI are 37.95 $\pm 20.27,69.48$ \pm 8.52 , and $10.96 \pm 8.45$, respectively (Table 2 ). High correlations are shown between the NRS and the DPPI $(r=0.809, \mathrm{p}<0.05)$ (Table 3$)$. 
The DPPI scale $(\mathrm{r}=0.437, \mathrm{p}<0.05)$ showed "moderate" significant correlations with the SF-36, while the NRS $(r=0.370, p<0.05)$ showed "weak" significant correlations (Table 3). Each item of the DPPI score is also significantly correlated with those of the SF-36 (Table 4). The DPPI-3 is moderately correlated with the SF-36 $(r=0.418, p<0.05)$, and the DPPI- 1 and the DPPI-2 are significantly correlated $(r=0.369$, $\mathrm{p}<0.05)(\mathrm{r}=0.391, \mathrm{p}<0.05)$ (Table 4$)$. There are no statistically significant correlations between the DPPI, the NRS score, and the BDI score $(p>0.05)$ (Table 4). An analysis of the correlations between each subsection of the DPPI and the SF-36 showed a statistically significant correlation; especially for "End-range pain" of DPPI-1 $(r=0.408$, $\mathrm{p}<0.05)$ and "Noon pain" of DPPI-2 $(\mathrm{r}=0.415, \mathrm{p}<0.05)$ shows a moderate correlation (Table 4). There are no statistically significant correlations between each subsection of the DPPI-2, the DPPI-3 score, and the BDI score ( $\mathrm{p}>0.05)$. "End-range pain" and BDI showed

Table 2. Mean \pm SD of subsections of DPPI scale

\begin{tabular}{llll}
\hline & \multicolumn{1}{c}{ Item } & \multicolumn{1}{c}{ Subsection } & Mean \pm SD \\
\hline DPPI & DPPI-1 & Starting range & $2.56 \pm 1.986$ \\
& & Mid-range & $2.92 \pm 2.120$ \\
& & End range & $3.33 \pm 2.255$ \\
& DPPI-2 & Morning (after sleep) & $1.65 \pm 1.766$ \\
& & Noon & $2.68 \pm 1.892$ \\
& NPPI-3 & Noucht (before sleep) & $2.41 \pm 2.185$ \\
& DPPI Total score & & $2.56 \pm 1.871$ \\
NRS & & & $18.7 \pm 10.834$ \\
SF-36 & & & $37.95 \pm 20.27$ \\
BDI & & & $69.48 \pm 8.517$ \\
\hline
\end{tabular}

DPPI: daily physical pain intensity, NRS: numerical rating scale, SF-36: ADL short form-36, BDI: beck depression inventory. a significant correlation $(\mathrm{r}=0.289, \mathrm{p}<0.05)($ Table 4$)$.

\section{DISCUSSION}

Pain was one of the most-common reasons that patients seek medical care. Given the strong interest in the development of treatments to effectively reduce pain, a pressing need for reliable metrics that can aid physicians in objective and accurate assessments of pain at baseline, and with the formulation of therapeutic responses both in terms of pain intensity and its impact on ADL, has emerged.$^{16}$ We investigated correlations between the DPPI scale and the NRS, and between the DPPI scale and the QOL (SF-36) and BDI questionnaires high correlations were shown between the NRS and the DPPI. The DPPI scale showed "moderate" significant correlations with the SF-36, while the NRS showed "weak" significant correlations. There were no statistically significant correlations between each subsection, however "End-range pain" and BDI showed a significant correlation.

The first step of the management of pain was the attainment of an assessment that was as valid as possible. ${ }^{17}$ Many residents had

Table 3. Correlations between DPPI, NRS score, and SF-36 score

\begin{tabular}{lcccc}
\hline & & NRS & SF-36 & BDI \\
\hline DPPI & $r$ & $0.809^{* *}$ & $-0.437^{\star *}$ & 0.199 \\
& $p$ & 0.001 & 0.001 & 0.318 \\
& $N$ & 73 & 73 & 73 \\
NRS & $\mathrm{N}$ & 1 & $-0.370^{* *}$ & 0.115 \\
& $\mathrm{p}$ & & 0.001 & 0.334 \\
& $\mathrm{~N}$ & 73 & 73 & 73 \\
\hline
\end{tabular}

${ }^{*}$ Correlation is significant at the 0.01 level (two-tailed). ${ }^{*}$ Correlation is significant at the 0.05 level (two-tailed). DPPI: daily physical pain intensity, NRS, numerical rating scale.

Table 4. Correlations between SF-36, BDI score, and subsections of DPPI scale

\begin{tabular}{|c|c|c|c|c|c|c|}
\hline & & & \multicolumn{2}{|c|}{ SF-36 } & \multicolumn{2}{|c|}{$\mathrm{BDI}$} \\
\hline & & & $r$ & $p$ & $r$ & $p$ \\
\hline \multirow[t]{9}{*}{ DPPI } & DPPI-1 & Total Score & $-0.369^{*}$ & 0.001 & 0.164 & 0.166 \\
\hline & & Starting range & -0.227 & 0.053 & 0.011 & 0.930 \\
\hline & & Mid-range & $-0.262^{*}$ & 0.025 & 0.086 & 0.470 \\
\hline & & End range & $-0.408^{* *}$ & 0.001 & $0.289^{*}$ & 0.013 \\
\hline & DPPI-2 & Total score & $-0.391^{*}$ & 0.001 & 0.061 & 0.608 \\
\hline & & Morning (after sleep) & $-0.320^{*}$ & 0.007 & 0.096 & 0.425 \\
\hline & & Noon & $-0.415^{\star \star}$ & 0.001 & 0.107 & 0.368 \\
\hline & & Night (before sleep) & $-0.338^{* *}$ & 0.003 & -0.006 & 0.961 \\
\hline & DPPI-3 & Touch & $-0.418^{\star \star}$ & 0.001 & 0.061 & 0.607 \\
\hline
\end{tabular}

${ }^{* *}$ Correlation is significant at the 0.01 level (two-tailed).

${ }^{*}$ Correlation is significant at the 0.05 level (two-tailed).

DPPI: daily physical pain intensity, SF-36: ADL short form-36, BDI: beck depression inventory. 
several diagnoses that was directly related to pain such as degenerative arthritis, diabetic neuropathy, and musculoskeletal disease. It had been shown that the more-effective management of patient pain through a systematic evaluation of pain intensity is associated with improved outcomes. ${ }^{18}$ In contrast, A poor assessment and untreated pain increased disability and decreased quality of life. ${ }^{19}$ The existence and intensity of pain were measured by the self-report of patient. Intensity scales were commonly used for measuring acute pain, and they included the NRS, the visual analog scale (VAS), and the Wong-Baker FACES Pain Rating Scale and its revised form. ${ }^{20}$ These scales were useful to gain an understanding of all of the degrees of the effects related to the pain. The major drawback of these assessment tools was that they measured only one pain dimension, as follows: severity. Virtually all of the assessments of pain were a variation of the question "What is your pain level?" A limitation of the assessment of the pain disturbed the diagnosis and produce an overly simplistic evaluation. ${ }^{21}$ In a case where the relief of pain was a result of treatment, the effect of the treatment for previous pain becomes complicated and obscured by the newly appeared pain. More than one pain assessment should therefore be considered to make an accurate decision about the effects of any pain intervention. ${ }^{21}$ The additional features of pain should inform the selection of a treatment regimen, and these included pain qualities, duration, impact on functional capabilities, and underlying causes. ${ }^{16}$ However other multidimensional assessments such as the full McGill pain questionnaire and the brief pain inventory typically required more time to administer. ${ }^{22}$ Therefore, we suggest the DPPI scale which is a simplistic evaluation including the quality of the pain and interference of ADL.

This was the first study to establish the concurrent validity of a new focus on ADL for the assessment of pain in patients. It was revealed that the natural course of the osteoporotic vertebral fracture resulted in severe long-lasting pain, disability, reduced ADL, and poor-health-related quality of life. ${ }^{23}$ NRS and visual analog scale (VAS) were intensity scales commonly used for measuring acute pain. ${ }^{24}$ The NRS required little training to administer and score and had been found to be acceptable to patients. ${ }^{25}$ The NRS was widely used due to its simplicity and adaptability to a broad range of populations and settings. Strengths of this measure over the pain NRS were the ability to be administered both verbally and in writing, as well as its simplicity of scoring. In this study, High correlations are shown between the NRS and the DPPI which means DPPI also a valid and reliable scale to measure pain intensity.

In this study, the correlation between the SF-36 and the DPPI scale was more significant than the relation between the SF-36 and the NRS. Because each item of the DPPI score was significantly correlated with those of the SF-36. The DPPI-3 was moderately correlated with the SF-36, and the DPPI-1 and the DPPI-2 were significantly correlated. Especially for "End-range pain" of DPPI-1, "Noon pain" of DPPI-2 and "Touch pain" of DPPI-3 showed a moderate correlation. End-range became even more blurred in conditions in which ROM losses were accompanied by pain and sensitization. ${ }^{26}$ In this conditions, the experience of pain and reduced tolerance to stretching determined the end-ranges. ${ }^{26}$ Loss of ROM affected activities and likely to be considered dysfunctional by person concerned. Actually, shoulder pain impaired a ability of patient to achieve independence regarding simple-to-complex daily activities. ${ }^{27}$ The influence of pain sensitivity i.e. "Touch pain” of DPPI-3 on the treatment outcomes was the critical concern. ${ }^{23}$ Many studies showed that the high relationship between pain sensitivity and physical activity. ${ }^{8}$ When the intensity and frequency of pain increased in patients with chronic pain, the patients became physically more sensitive and they direct their attention to pain-related fear and other catastrophic thoughts. ${ }^{28}$ "Noon" defined the peak of activity in the day. Noon pain directly linked with activity and QOL. ${ }^{29}$ Therefore each score of end-range pain, Noon pain and touch pain of DPPI influenced this result of significantly correlation between the SF-36 and the DPPI scale more than the relation between the SF-36 and the NRS.

Several self-report-measurements had been designed to assess anxiety disorders. The scale was the Penn state worry questionnaire for generalized anxiety disorder and the mobility inventory for agoraphobia. ${ }^{30}$ Bair MJ reported that psychopathology (i.e., depression) was associated with poor pain-related outcomes such as elevated pain intensity, functional limitations, and non-recovery. ${ }^{31}$ According to the results of this paper, there was no statistically significant correlation between the DPPI scale and the depression questionnaire (BDI). Over the last 30 years, many studies had focused on the relationship between pain and depressive illness. However, there was evidence that chronic and not severe pain was not specific to depression. ${ }^{32}$ In a recent study evidence was presented that between lumbar pain and depression score were showed just a weak relation degrees. ${ }^{33}$ Depressive symptoms were associated with increased hospital stays, fewer functional improvements, 
increased medical complications and poor social integration..$^{34}$ Because of the subject of this study had mild and chronic pain with NRS 37 points out of 100, not affect their emotional conditions.

With the DPPI scale, pain was closely monitored, and the transfer phase of pain according to the three fields of movement, persistence, and sensitivity was confirmed. Especially, it was possible to classify the patient who had pain of a low intensity that persisted all-day long, and it therefore leads to distress that was reflected on the QOL. The digitized DPPI score was shown as a triangle, and if each aspect of pain was severe, the triangle become bigger. The shape of the triangle and the change of an aspect can provide information for the prediction of disease for which accumulated DPPI data are used. Future research may investigate the various disease pains of hospital patients who can expect QOL changes that are due to pain and disorder. The development of more-comprehensive tools for patient assessment is the first step in the achievement of the ultimate goal of effective acute-pain management. The results of this study show promise for the development of an ADL-focused tool for an assessment of the subjective pain of patients that is more objective, and this will be the basis of the authors' ongoing research.

\section{ACKNOWLEDGEMENTS}

This work was supported by National Research Foundation of Korea Grant funded by the Korean Government (NRF-2016R1D1A3B03932586).

\section{REFERENCES}

1. Classification of chronic pain. Descriptions of chronic pain syndromes and definitions of pain terms. Prepared by the International Association for the Study of Pain. Subcommittee on Taxonomy. Pain Suppl. 1986;3: S1-226.

2. Bair MJ, Robinson RL, Katon W et al. Depression and pain comorbidity: a literature review. Arch Intern Med. 2003;163(20):2433-45.

3. Bartynski WS. Clinical, anatomic, and imaging correlation in spine-related pain: the essential elements. Tech Vasc Interv Radiol. 2009;12(1):2-10.

4. Bigliani LU, Kelkar R, Flatow EL et al. Glenohumeral stability. Biomechanical properties of passive and active stabilizers. Clin Orthop Relat Res. 1996(330):13-30.

5. Chanques G, Jaber S, Barbotte E et al. Impact of systematic evaluation of pain and agitation in an intensive care unit. Crit Care Med. 2006;34(6): 1691-9.

6. Dunwoody CJ, Krenzischek DA, Pasero C et al. Assessment, physiological monitoring, and consequences of inadequately treated acute pain.
Pain Manag Nurs. 2008;9(1 Suppl):S11-21.

7. Eccleston C, Crombez G. Pain demands attention: a cognitive-affective model of the interruptive function of pain. Psychol Bull. 1999;125(3): 356-66.

8. Ellingson LD, Colbert LH, Cook DB. Physical activity is related to pain sensitivity in healthy women. Med Sci Sports Exerc. 2012;44(7):1401-6.

9. Feuerstein M, Beattie P. Biobehavioral factors affecting pain and disability in low back pain: mechanisms and assessment. Phys Ther. 1995;75(4): 267-80.

10. Field T, Diego M, Gonzalez G, Funk CG. Knee arthritis pain is reduced and range of motion is increased following moderate pressure massage therapy. Complement Ther Clin Pract. 2015;21(4):233-7.

11. Gélinas C, Puntillo KA, Joffe AM et al. A Validated approach to evaluating psychometric properties of pain assessment tools for use in nonverbal critically ill adults. Semin Respir Crit Care Med. 2013;34(02):153-68.

12. Gronblad M, Jarvinen E, Airaksinen O et al. Relationship of subjective disability with pain intensity, pain duration, pain location, and work-related factors in non-operated patients with chronic low back pain. Clin J Pain. 1996;12(3):194-200.

13. Hawker GA, Mian S, Kendzerska T et al. Measures of adult pain: visual analog scale for pain (VAS pain), Numeric Rating Scale for Pain (NRS Pain), McGill Pain Questionnaire (MPQ), short-form McGill pain questionnaire (SF-MPQ), chronic pain grade scale (CPGS), short form-36 bodily pain scale (SF-36 BPS), and measure of intermittent and constant osteoarthritis pain (ICOAP). Arthritis Care Res (Hoboken). 2011;63(11 Suppl):S240-52.

14. Herr KA, Mobily PR. Complexities of pain assessment in the elderly. Clinical considerations. J Gerontol Nurs. 1991;17(4):12-9.

15. Ista E, van Dijk M, van Achterberg T. Do implementation strategies increase adherence to pain assessment in hospitals?: a systematic review. Int J Nurs Stud. 2013;50(4):552-68.

16. Kim HJ, Suh BG, Lee DB et al. The influence of pain sensitivity on the symptom severity in patients with lumbar spinal stenosis. Pain Physician. 2013;16(2):135-44.

17. Krebs EE, Carey TS, Weinberger M. Accuracy of the pain numeric rating scale as a screening test in primary care. J Gen Intern Med. 2007; 22(10):1453-8.

18. Johnston CC, Gagnon A, Rennick J et al. One-on-one coaching to improve pain assessment and management practices of pediatric nurses. J Pediatr Nurs. 2007;22(6):467-78.

19. LEEDUIK. Textbook of pain medicine. 4 ed. The Korean Pain Society. 2012.

20. Lippitt SB, Vanderhooft JE, Harris SL et al. Glenohumeral stability from concavity-compression: a quantitative analysis. J Shoulder Elbow Surg. 1993;2(1):27-35.

21. McWilliams LA, Goodwin RD, Cox BJ. Depression and anxiety associated with three pain conditions: results from a nationally representative sample. Pain. 2004;111(1-2):77-83.

22. Melzack R. The McGill pain questionnaire: major properties and scoring methods. Pain. 1975;1(3):277-99.

23. Radcliff KE, Rihn J, Hilibrand A et al. Does the duration of symptoms in patients with spinal stenosis and degenerative spondylolisthesis affect outcomes?: analysis of the spine outcomes research trial. Spine (Phila Pa 1976). 2011;36(25):2197-210. 
24. Krebs EE, Carey TS, Weinberger M. Accuracy of the pain numeric rating scale as a screening test in primary care. J Gen Intern Med. 2007; 22(10):1453-8.

25. Huskisson EC, Wojtulewski JA, Berry H et al. Treatment of rheumatoid arthritis with fenoprofen: comparison with aspirin. Br Med J 1974;1: 176-80.

26. Sullivan MS, Shoaf LD, Riddle DL. The relationship of lumbar flexion to disability in patients with low back pain. Phys Ther. 2000;80(3):240-50.

27. Kim O. Sex differences in social support, loneliness, and depression among Korean college students. Psychol Rep. 2001;88(2):521-6.

28. Lim KB, Kim JY, Lee HJ et al. The relations among pain, emotional and cognitive-behavioral factors in chronic musculoskeletal pain patients. J vKorean Acad Rehabil Med. 2008;32(4):424-9.

29. Tang NK, Sanborn AN. Better quality sleep promotes daytime physical activity in patients with chronic pain? A multilevel analysis of the withinperson relationship. PLoS One. 2014;9(3):e92158.
30. Meyer TJ, Miller ML, Metzger RL et al. Development and validation of the Penn state worry questionnaire. Behav Res Ther. 1990;28(6):487-95.

31. Radnovich R, Chapman CR, Gudin JA et al. Acute pain: effective management requires comprehensive assessment. Postgrad Med. 2014; 126(4):59-72

32. Breslau N, Davis GC. Migraine, major depression and panic disorder: a prospective epidemiologic study of young adults. Cephalalgia. 1992; 12(2):85-90.

33. You JE, Jung EH. The effects of traditional physical therapy on pain reduction and depression level of patients with chronic low back pain. J Kor Soc Phys Ther. 2001;13(3):677-83

34. Smarr KL, Keefer AL. Measures of depression and depressive symptoms: beck depression inventory-II (BDI-II), center for epidemiologic studies depression scale (CES-D), geriatric depression scale (GDS), hospital anxiety and depression scale (HADS), and patient health questionnaire-9 (PHQ-9). Arthritis Care Res (Hoboken). 2011;63(11 Suppl):S454-66. 